\title{
Influence of pelvic asymmetry and idiopathic scoliosis in adolescents on postural balance during sitting
}

\author{
Ji-Yong Jung ${ }^{\mathrm{a}}$, Eun-Jong Cha ${ }^{\mathrm{b}}$, Kyung-Ah Kim ${ }^{\mathrm{b}}$, Yonggwan Won ${ }^{\mathrm{c}}$, Soo-Kyung Bok ${ }^{\mathrm{d}}$, Bong- \\ $\mathrm{Ok} \mathrm{Kim}^{\mathrm{d}}$ and Jung-Ja Kim ${ }^{\mathrm{e}, \mathrm{f}^{*}}$ \\ ${ }^{a}$ Department of Healthcare Engineering, Chonbuk National University, 567 Baekje-daero, Deokjin-gu, \\ Jeonju-si, Jeollabuk-do 561-756, South Korea \\ ${ }^{b}$ Department of Biomedical Engineering, College of Medicine, Chungbuk National University, 52 \\ Naesudong-ro, Seowon-gu, Cheongju, Chungbuk 361-763, South Korea \\ ${ }^{c}$ Department of Electronics and Computer Engineering, Chonnam National University, 77 Yongbong- \\ ro, Buk-gu, Gwangju 500-757, South Korea \\ ${ }^{d}$ Department of Rehabilitation Medicine, Chungnam National University Hospital, 220 Gung-dong, \\ Yuseong-gu, Daejeon-si, Chungchungnam-do 301-721, South Korea \\ ${ }^{e}$ Division of Biomedical Engineering, Chonbuk National University, 567 Baekje-daero, Deokjin-gu, \\ Jeonju-si, Jeollabuk-do 561-756, South Korea \\ ${ }^{f}$ Research Center of Healthcare \& Welfare Instrument for the Aged, Chonbuk National University, 567 \\ Baekje-daero, Deokjin-gu, Jeonju-si, Jeollabuk-do 561-756, South Korea
}

\begin{abstract}
The effects of pelvic asymmetry and idiopathic scoliosis on postural balance during sitting were studied by measuring inclination angles, pressure distribution, and electromyography. Participants were classified into a control group, pelvic asymmetry group, scoliosis group, and scoliosis with pelvic asymmetry and then performed anterior, posterior, left, and right pelvic tilting while sitting on the unstable board for 5 seconds to assess their postural balance. Inclination and obliquity angles between the groups were measured by an accelerometer located on the unstable board. Pressure distribution (maximum force and peak pressure) was analyzed using a capacitive seat sensor. In addition, surface electrodes were attached to the abdominal and erector spinae muscles of each participant. Inclination and obliquity angles increased more asymmetrically in participants with both pelvic asymmetry and scoliosis than with pelvic asymmetry or scoliosis alone. Maximum forces and peak pressures of each group showed an asymmetrical pressure distribution caused by the difference in height between the left and right pelvis and curve type of the patients' spines when performing anterior, posterior, left, and right pelvic tilting while sitting. Muscle contraction patterns of external oblique, thoracic erector spinae, lumbar erector spinae, and lumbar multifidus muscles may be influenced by spine curve type and region of idiopathic scoliosis. Asymmetrical muscle activities were observed on the convex side of scoliotic patients and these muscle activity patterns were changed by the pelvic asymmetry. From these results, it was confirmed that pelvic asymmetry and idiopathic scoliosis cause postural asymmetry, unequal weight distribution, and muscular imbalance during sitting.
\end{abstract}

Keywords: Pelvic asymmetry, adolescent idiopathic scoliosis, postural balance, sitting

\footnotetext{
*Address for correspondence: Jung-Ja Kim, Division of Biomedical Engineering, Chonbuk National University, 567 Baekje-daero, Deokjin-gu, Jeonju-si, Jeollabuk-do 561-756, South Korea. Tel.: +82-63-270-4102; Fax: +82-63-270-2247; Email: jungjakim@jbnu.ac.kr.
} 


\section{Introduction}

Idiopathic scoliosis is an abnormal curvature of the spine in the coronal plane. It has been reported that approximately $4 \%$ of adolescents between the ages of 10 and 18 have idiopathic scoliosis [1-3]. However, its pathogenesis remains unknown. Seze and Cugy [4] identified existing pathogenetic hypotheses by categorizing them within intrinsic and extrinsic factors and suggested the initial asymmetry of the body during growth phases may affect progression of adolescent idiopathic scoliosis (AIS). Aggravated scoliosis due to the effect of initial asymmetry of the spine has been associated with leg length inequality which can cause pelvic obliquity and tilting [5-7]. Previous studies discovered that asymmetrical leg lengths and abnormal curvature of the spine leads to altered trunk motion, postural control problems, muscle imbalance, and lesser stability than healthy subjects. Gerney [8] suggested leg length discrepancy leads to increasing several muscle activity and postural sway while quiet standing. Al-Eisa, et al. [9] confirmed that pelvic inequality alters the patterns of trunk movement and mechanics of the spine. Bussey [10] reported asymmetrical loading related flexed and rotated trunk motion can influence greater prevalence of pelvic asymmetry from the results in previous studies. Nault, et al. [11] and Sahli, et al. [12] identified decreased postural stability associated with spinal deformity during standing. Odermatt, et al. [13] found differences in muscle activity between the concave and convex sides of the spine by assessing electromyography (EMG) signals in the trunk muscles of AIS patients.

Generally, curve types of scoliosis are classified as C-shaped and S-shaped based on the direction and location of spinal deformity [14]. C-shaped and S-shaped curves refer to a single and double curve, respectively, in the thoracic, lumbar, or thoracolumbar region. These curve types are connected to asymmetrical rotation, elevation, and tilting of the pelvis which can cause various pain-related symptoms. Timgren and Soinila [15] investigated the association between leg length differences, pelvic asymmetry, and scoliosis based on clinical examination results and concluded that asymmetrical postural balance was correlated with convexity and concavity of the spinal curve. Shamberger [16] described the connection between asymmetrical alignment of the lower extremities and compensatory curvatures of the spine.

It is crucial to investigate the correlation between pelvic asymmetry and scoliosis in adolescents to prevent progression of the deformity because asymmetrical structure of the pelvis with abnormal curvature of the spine plays a fundamental role in the development of scoliosis. Idiopathic scoliosis occurs during adolescence, and adult scoliosis (degenerative scoliosis) is thought to be directly associated with untreated idiopathic scoliosis [14]. There has been extensive research on balance problems and abnormal muscle contractions on the convex side of the spine during standing and walking; however, there is no known study on how pelvic inequality affects postural balance, body pressure, and muscle function of patients with adolescent idiopathic scoliosis during sitting.

The purpose of this study was to determine the influence of pelvic asymmetry and idiopathic scoliosis in adolescents on postural balance by measuring inclination angles, pressure distribution, and muscle activation patterns in the frontal and sagittal planes during sitting. 


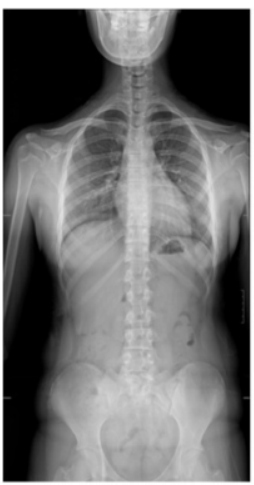

(a)

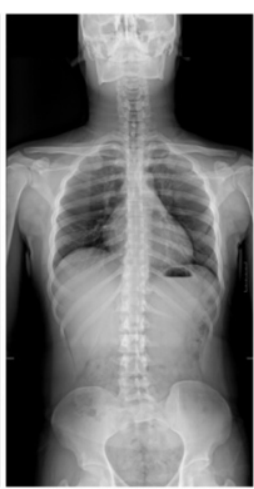

(b)

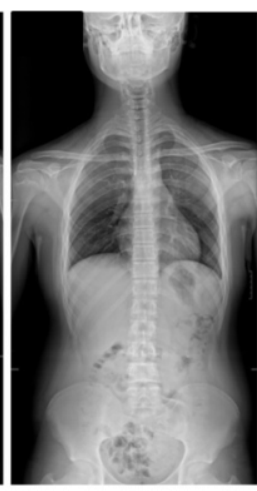

(c)

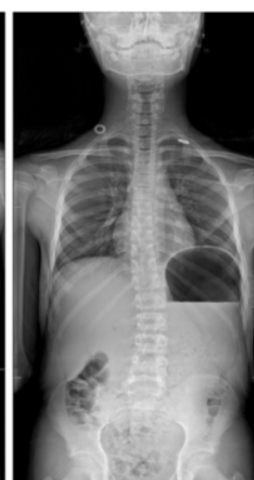

(d)

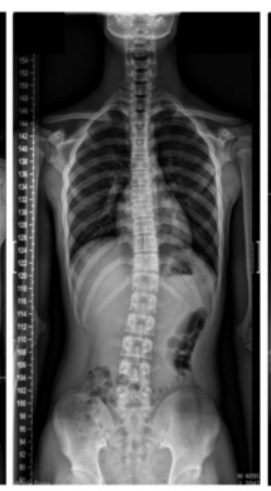

(e)

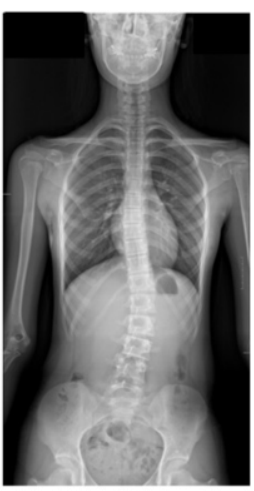

(f)

Fig. 1. (a) Control group; (b) asymmetry on the left side group; (c) asymmetry on the right side group; (d) scoliosis group; (e) scoliosis with asymmetry on the left side group; (f) scoliosis with asymmetry on the right side group.

\section{Experimental methods}

\subsection{Subjects}

The 32 subjects who participated in this study were recruited from the Department of Rehabilitation Medicine of Chungnam National University Hospital in Daejeon, South Korea. All subjects and their parents provided written informed consent prior to their voluntary participation. Subjects were classified into six groups, as shown in Figure 1. The control group (CG) consisted of 8 adolescents with normal spinal $\mathrm{X}$-rays and no previous spinal disorders. The asymmetry-on-the-left-pelvis group (ALPG) consisted of 6 adolescents with increased height of the left pelvis and a difference of length between the legs of $12.09 \pm 4.39 \mathrm{~mm}$. The asymmetry-on-the-right-pelvis group (ARPG) consisted of 6 adolescents with increased height of the right pelvis and a difference of length between the legs of $8.52 \pm 1.73 \mathrm{~mm}$. The scoliosis group (SG) consisted of 6 adolescents with C-shaped lumbar or thoracolumbar curves to the left convex side defined by a cobb angle between $10^{\circ}$ and $19^{\circ}$ and no previous conservative or surgical treatment for the scoliosis. The scoliosis-with-asymmetry-on-theleft-pelvis group (SALPG) and scoliosis-with-asymmetry-on-the-right-pelvis group (SARPG) consisted of 6 adolescents with C-shaped curves ranging from $10^{\circ}$ to $21^{\circ}$ to the left convex side and a difference of height of the pelvis of $7.08 \pm 1.18 \mathrm{~mm}$ and $10.14 \pm 4.42$, respectively. The mean age, height, and body weight of the participants was $13.57 \pm 1.65$ years, $146.1 \pm 6.23 \mathrm{~cm}$, and $47.67 \pm 5.75 \mathrm{~kg}$, respectively.

\subsection{Experimental instruments}

Inclination and obliquity angles between the groups were assessed by using an unstable board in the shape of a hemisphere. This board consisted of an accelerometer and photo sensors for detecting inclination angles in the frontal and sagittal planes. The curvature radius structure of this board was designed to incline in the anterior, posterior, left, and right direction according to pelvic movements [17]. The accuracy and reliability of the results from this board were confirmed in a previous study [18]. 


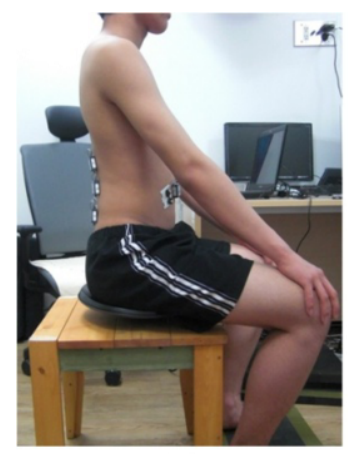

(a)

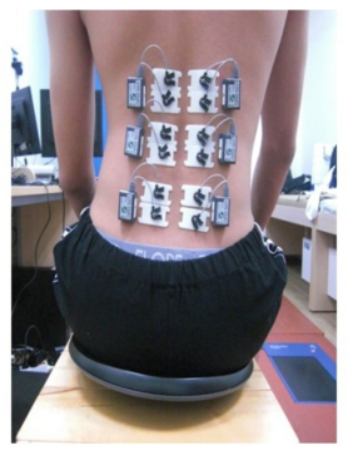

(b)

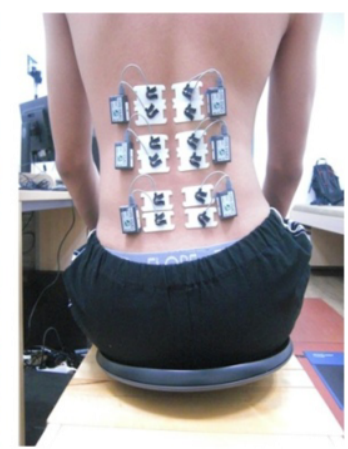

(c)

Fig. 2. (a) Anterior and posterior pelvic tilting; (b) lateral pelvic tilting (right side); (c) lateral pelvic tilting (left side).

Differences in pressure distribution among each group during sitting was measured by a Pliance seat sensor system (Novel Gmbh., Munich, Germany), which contained 256 capacitive sensors, sampled at a rate of $50 \mathrm{~Hz}$, in a $16 \times 16$ matrix. Data were transferred using a Bluetooth connection and then recorded on a computer.

Electromyography (EMG) signals were recorded using an 8-channel wireless Noraxson Telemyo 2400T System (Noraxson Inc., Scottsdale, USA). Surface electrodes were attached to the trunk muscles (external oblique, thoracic erector spinae, lumbar erector spinae, and lumbar multifidus muscles) bilaterally after shaving the skin in the corresponding area and cleansing it with alcohol to reduce skin resistance $[19,20]$.

\subsection{Procedure}

All subjects were instructed to perform anterior, posterior, left, and right pelvic tilt with their arms crossed on the contra-lateral shoulder during sitting on the unstable board to measure their postural balance, pressure distribution, and muscle activity pattern. The unstable board was located in the center of a stool which was used to remove the influence of structure and material of the chair on pelvic movements. Additionally, a foot support was employed to adjust the knee and ankle angle at $90^{\circ}$ to prevent leg movements from effecting pelvic tilting. Subjects kept the anterior, posterior, and lateral pelvic tilt pose for 5 seconds and then took a 5 minute rest between experiments, as shown in Figure 2.

\subsection{Data analysis}

Pelvic inclination and obliquity angles according to pelvic tilting in the frontal and sagittal planes were analyzed at $100 \mathrm{~Hz}$ sampling rate by using a program written in LabVIEW (National Instruments Co., Texas, USA). Pressure distribution data were subdivided into two regions of masks (left and right sides) due to the structure of the unstable board. These data were analyzed for maximum force and peak pressure by using Novel software (Novel Gmbh., Munich, Germany). MyoResearch Master XP 1.07 (Noraxson Inc., Scottsdale, USA) was used to analyze the contractions of the trunk muscles. All EMG signals were amplified, bandpass filtered (passband 20-450 Hz), notch-filtered at $60 \mathrm{~Hz}$, and then sampled at $1000 \mathrm{~Hz}$. To normalize the difference of the magnitude of muscle contractions, it was expressed as a percentage relative to the maximum voluntary contraction (MVC). Two MVC trials were conducted to obtain the maximal EMG of abdominal (external oblique) and erector spinae 
(thoracic erector spinae, lumbar erector spinae, and lumbar multifidus) muscles. Subjects were asked to lift their upper trunk from the floor for maximal isometric extension and flexion for five seconds in three measurements, respectively. An assistant on the anterior side of the patients applied an external load to the chest and scapulae, and a second assistant held the participant's ankles to stabilize the trunk position [21].

Statistical analysis was performed using SPSS PASW statistics 18 (SPSS Inc., Chicago, USA). A ttest was used to examine the differences in inclination angle, pressure distribution, and muscle activation patterns between anterior, posterior, left, and right, at the $\mathrm{p}<0.05$ level. Comparisons were also made for measured variables in experimental results between the groups by using one-way ANOVA with Post Hoc Scheffé test, at the $\mathrm{p}<0.05$ level.

\section{Results}

\subsection{Inclination and obliquity angle}

Figure 3 illustrates the inclination and obliquity angles between the groups according to anterior, posterior, left, and right pelvic tilting. As compared with inclination angles among CG, ALPG, ARPG, and SG, the angle results of ALPG, ARPG, and SG were tilted significantly to the posterior when performing anterior and posterior tilting during sitting $(\mathrm{p}<0.05)$. In contrast, there were significant increases in inclination angles to the anterior in the SALPG and SARPG $(\mathrm{p}<0.05)$. During left and right pelvic tilting, the obliquity angles in the ARPG, SG, SALPG, and SARPG increased significantly on the left side, while lateral tilting angles of the ALPG decreased significantly on the left side ( $\mathrm{p}<$ 0.05 and $\mathrm{p}<0.05$, respectively). In addition, there were significant differences in angle variations between the ALPG, SALPG and SARPG when performing anterior and left pelvic tilts during sitting $(\mathrm{p}<0.05)$.

\subsection{Pressure distribution}

Comparisons of pressure distribution between the groups are presented in Figure 4. Maximum forces and peak pressures of the ALPG and SALPG increased on the right side, while pressure distribution of the ARPG, SG, and SARPG decreased on the right side when performing lateral pelvic tilting during sitting. There were no significant differences in pressure distribution between the six groups.
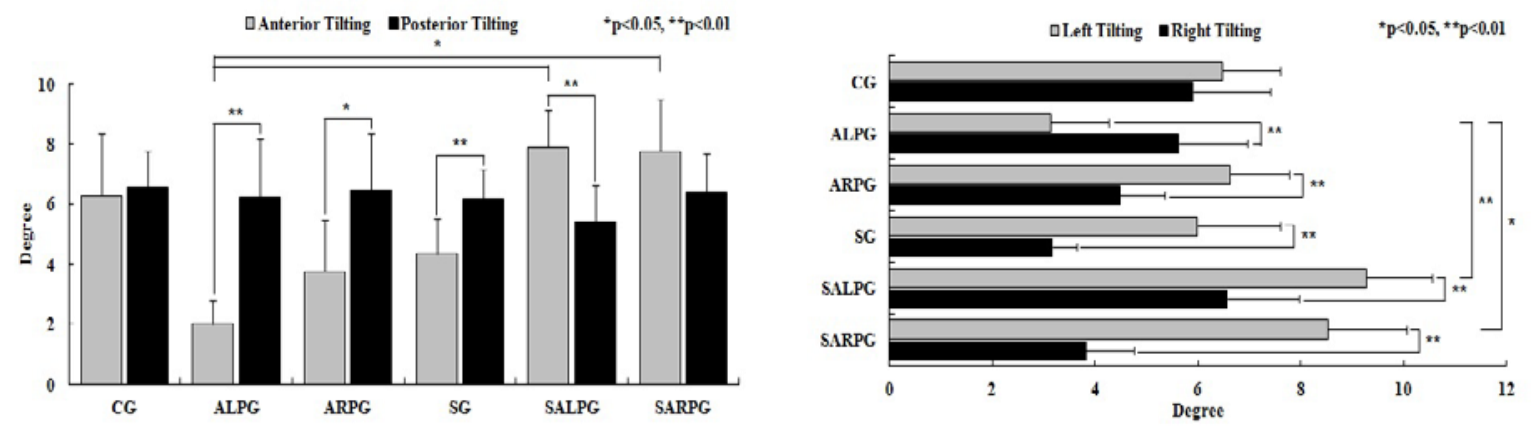

Fig. 3. Differences in inclination and obliquity angles between the groups with pelvic anterior, posterior, left, and right tilting. 

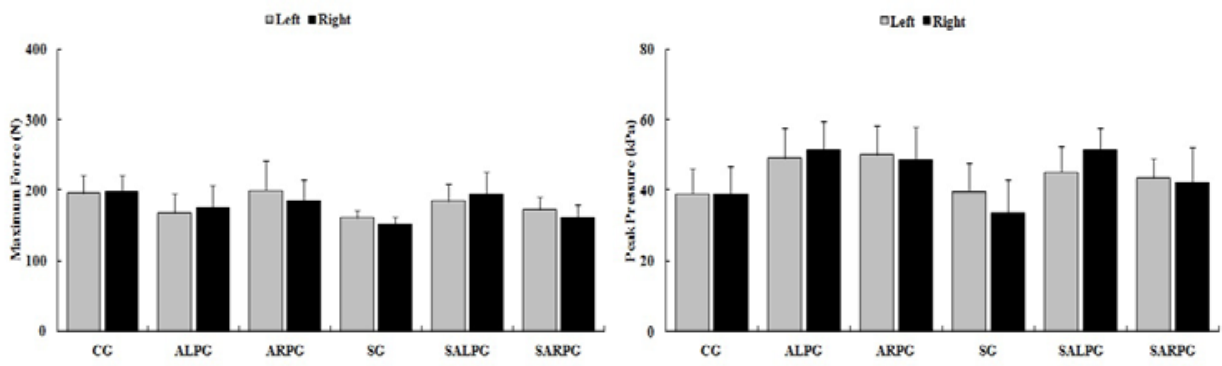

Fig. 4. Comparisons of maximum force and peak pressure between the groups.

\subsection{Muscle activity}

Differences in external oblique and thoracic erector spinae muscle activities between the left and right sides during anterior, posterior, left, and right tilting are presented in Figures 5 and 6 . In external oblique muscles, the muscle activities of the ALPG and SALPG were less on the right side than the left side, while the muscle activities of the ARPG, SG, and SARPG increased on the right side when performing anterior, posterior, left, and right tilting during sitting. There were no significant differences in the muscle activities between both sides or between the groups. In terms of thoracic erector spinae muscles, muscle activities of the ALPG, SG, and SALPG increased on the left side, whereas the opposite tendency was observed in the ARPG and SARPG. In particular, there were significant differences in muscle activities between the left and right sides of the thoracic erector spinae muscles in the ALPG (right tilting), ARPG (left tilting), SG (right tilting), SALPG (anterior, posterior, left, and right tilting), and SARPG (left and right tilting) $(\mathrm{p}<0.05)$.
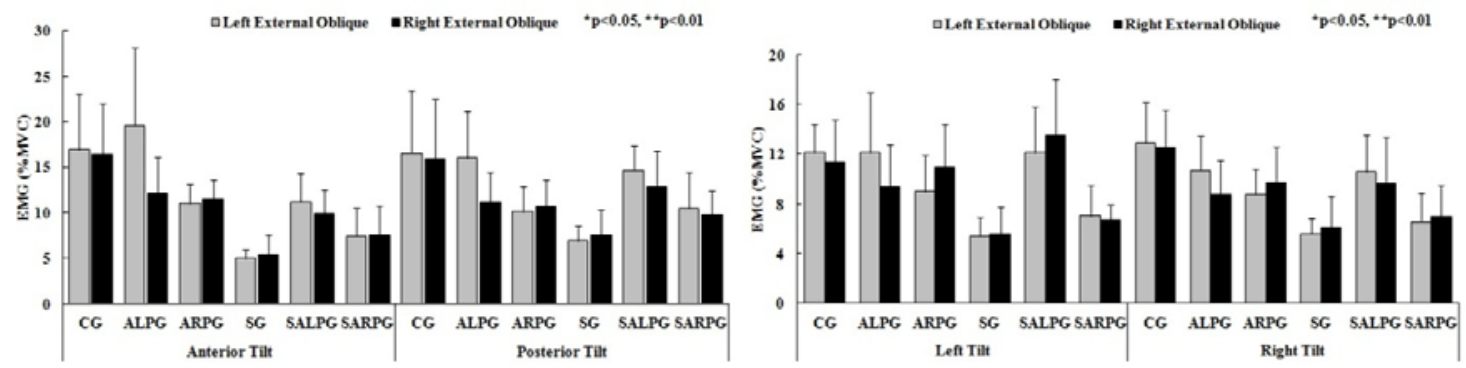

Fig. 5. Differences in external oblique muscle activity between the groups.
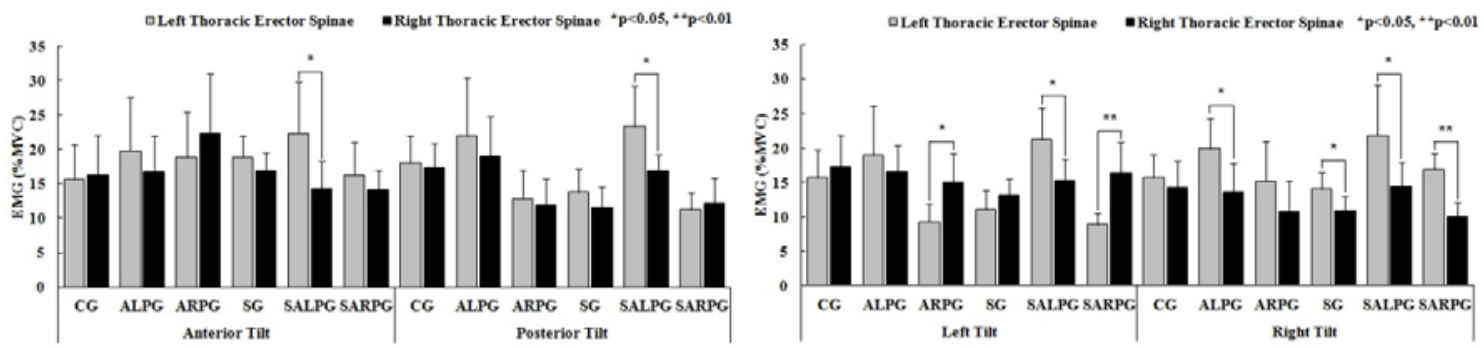

Fig. 6. Differences in thoracic erector spinae muscle activity between the groups. 

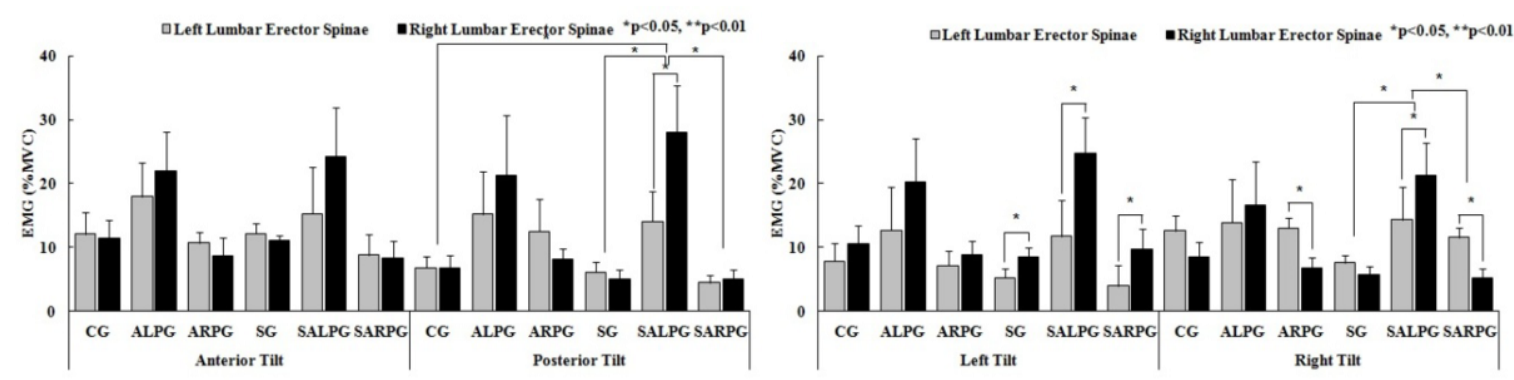

Fig. 7. Differences in lumbar erector spinae muscle activity between the groups.
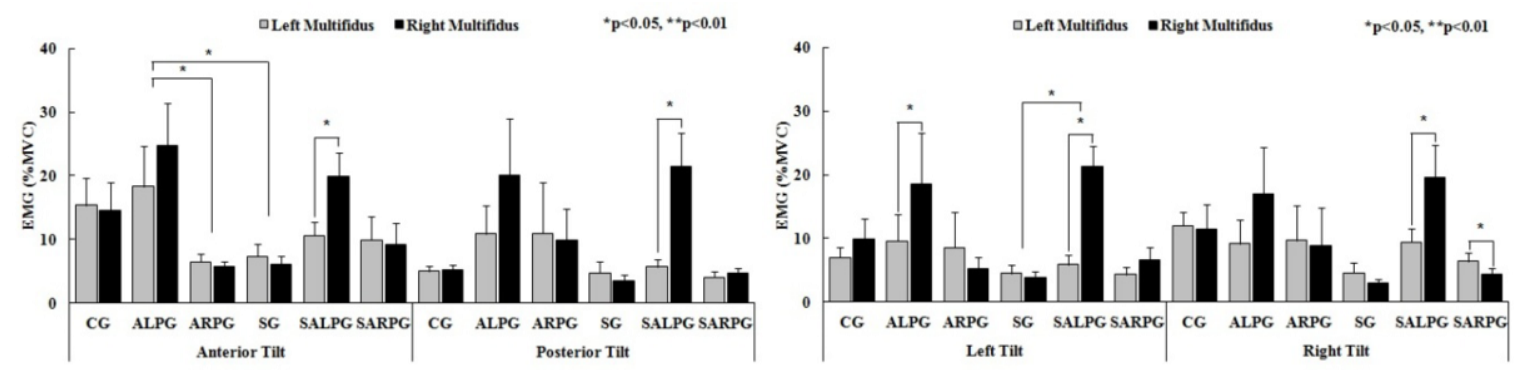

Fig. 8. Differences in lumbar multifidus muscle activity between the groups.

In contrast, muscle activity patterns of the lumbar erector spinae and lumbar multifidus muscles were different from the results of the external oblique and thoracic erector spinae muscle activities, as shown in Figures 7 and 8. In the lumbar erector spinae muscles, the muscle activities of the ALPG and SALPG increased on the right side more than the left side, while muscle activities of the ARPG, SG, and SARPG decreased on the right side when performing pelvic tilting during sitting. There were significant differences in muscle activity between the left and right sides of the lumbar erector spinae muscles in the ARPG (right tilting), SG (left tilting), SALPG (posterior, left, and right tilting), and SARPG (left and right tilting) ( $\mathrm{p}<0.05$ ). In addition, significant differences in muscle activities between the groups were observed in the right side of lumbar erector spinae muscles when performing posterior and right pelvic tilting during sitting $(\mathrm{p}<0.05)$. In terms of lumbar multifidus muscles, the muscle activities of the ALPG and SALPG were reduced on the left side, whereas muscle activities of the ARPG, SG, and SARPG increased on the left side. There were significant differences in muscle activities between the left and right sides of the lumbar multifidus muscles in the ALPG (left tilting), SALPG (anterior, posterior, left, and right tilting), and SARPG (right tilting) $(\mathrm{p}<0.05)$. Furthermore, significant differences in muscle activities between the groups were found on the right side of the lumbar multifidus muscles when performing anterior and left tilting during sitting $(\mathrm{p}<0.05)$.

\section{Discussion}

In this study, the inclination angles, pressure distribution, and muscle activities among the CG, ALPG, ARPG, SG, SALPG, and SARPG were assessed when the participants performed anterior, posterior, left, and right pelvic tilting while in the seated position. 
The ALPG, ARPG, and SG showed more tilted asymmetry angles to the posterior side than the anterior side during sitting. In contrast, the inclination angles of the SALPG and SARPG increased anteriorly compared to that of the CG, ALPG, ARPG, and SG. In the case of left and right pelvic tilting during sitting, the obliquity angles of the ALPG, SG, SALPG, and SARPG increased on the left side, while the angle variation of the ARPG decreased on the left side compared to the obliquity angle of the CG. Friberg [22] and Gofton [23] suggested that length asymmetry may affect pelvis deformation in the frontal and sagittal planes, and may negatively influence sitting posture in compensation. From the results of the study, it was confirmed that pelvic asymmetry and scoliosis can affect postural balance in the frontal and sagittal planes during sitting, and postural asymmetry increased in participants with both pelvic asymmetry and scoliosis more than pelvic asymmetry or scoliosis alone.

It has been reported that unbalanced sitting can cause pressure ulcers and advance deformation of the spine [24]. In this study, maximum forces and peak pressures of the subjects showed an asymmetrical pressure distribution caused by pelvic asymmetry and idiopathic scoliosis. However, there were no significant differences in the pressure distribution between the six groups. Bruyneel and Mesure [25] argued that AIS patients are in a learning situation during mediolateral destabilization in the seated position. This adaptability of dynamic strategies to stabilize the posture between the tests was more stable in the second and third tests than in the first test, and so body mass of the scoliosis patients was redistributed with no significant differences during dynamic sitting. On the other hand, Wang, et al. [26] reported the pelvic position in scoliosis patients could be associated with spinal alignment and sitting balance due to the lumbar spine being connected to the pelvis, which plays an important role in transferring the load. Therefore, the pressure distribution patterns of patients with scoliosis and pelvic asymmetry were changed by the asymmetry of the left pelvic. Additionally, there was no significant difference in the pressure distribution due to the correlation between the spine and the pelvis. This study's finding supported the hypothesis that differences in the height of the pelvis between the left and right sides and C-shaped curves with left convex sides can influence the pressure distribution during sitting.

Trunk muscle activities when performing anterior, posterior, left, and right tilting during sitting were analyzed to evaluate the correlation between pelvic asymmetry and scoliosis. Muscle activities of the external oblique muscles were different between pelvic asymmetry patients and patients with both scoliosis and pelvic asymmetry. In addition, there were only muscle contraction pattern changes in the SARPG compared to the ARPG. This meant that the curve direction in scoliosis patients may affect the muscle activities of the abdominal muscles during sitting. Odermatt, et al. [13] and Cheung, et al. [27] demonstrated that larger muscle activities have often been observed on the convex side. Increased thoracic erector muscle activities according to the direction of asymmetry in the pelvis and the convex side of the spine were observed in patients with both pelvic asymmetry and scoliosis during sitting. In contrast, asymmetrical muscle activation appeared in the lumbar erector spinae and lumbar multifidus muscles of the ALPG, ARPG, SALPG, and SARPG, regardless of the direction of pelvic asymmetry and scoliosis. Differences in muscle activity pattern in subjects with pelvic asymmetry and scoliosis between thoracic erector spinae, lumbar erector spinae, and lumbar multifidus muscles may be influenced by the curve type and region of scoliosis and by compensation strategies of the body to keep the trunk balanced during sitting. Mahaudens, et al. [28] demonstrated prolonged activation of quadrates lumborum and erector spinae muscles and the co-contractions between the spinal muscles on the convex side to stabilize the spine. In addition, adolescent idiopathic scoliosis patients have prolonged activities of erector spinae muscles, which cause structural bony spinal deformities such as pelvic asymmetry [29]. 


\section{Conclusion}

The effects of pelvic asymmetry and idiopathic scoliosis in adolescents on postural balance were evaluated by measuring inclination angles, pressure distribution, and muscle activation patterns in the frontal and sagittal planes. The results obtained in this study indicated that pelvic asymmetry and idiopathic scoliosis in adolescents can affect the postural balance in the frontal and sagittal planes during sitting and can compound asymmetry in pelvic asymmetry with idiopathic scoliosis. Also, differences in the height of the pelvis, as well as region and direction of the scoliosis, can influence the unbalanced sitting pressure distribution and prolong the asymmetrical muscle activity patterns of external oblique, thoracic erector spinae, lumbar erector spinae, and lumbar multifidus muscles. Consequently, it was concluded that pelvic asymmetry and idiopathic scoliosis can have a significant effect on postural balance, pressure distribution, and trunk muscle activity.

Furthermore, this paper suggests that a structure of unstable board may be utilized to assess the effects of asymmetrical postural balance and muscle activity caused by pelvic asymmetry and scoliosis on the physical activities and exercise capabilities of adolescent patients in their daily lives.

\section{Acknowledgment}

This work was supported by the National Research Foundation of Korea (NRF) grant funded by the Korea government (MSIP) (NRF-2013R1A2A2A04016782).

\section{References}

[1] D. Wang, L. Shi, W.C. Chu, R.G. Burwell, J.C. Cheng and A.T. Ahuja, Abnormal cerebral cortical thinning pattern in adolescent girls with idiopathic scoliosis, Neuroimage 59 (2012), 935-942.

[2] A. Sud and A. Tsirikos, Current concepts and controversies on adolescent idiopathic scoliosis: Part I, Indian Journal of Orthopaedics 47 (2013), 117-128.

[3] C.W.J. Cheung, S.Y. Law and Y.P. Zheng, Development of 3-D ultrasound system for assessment of adolescent idiopathic scoliosis (AIS) and system validation, Proceeding of 35th Annual International Conference IEEE Engineering in Medicine and Biology Society (EMBC) 2013, 3-7.

[4] M. Seze and E. Cugy, Pathogenesis of idiopathic scoliosis: A review, Annals of Physical Rehabilitation Medicine 55 (2012), 128-138

[5] J.W. Raczkowski, B. Daniszewska and K. Zolynski, Functional scoliosis caused by leg length discrepancy, Archives of Medical Science 6 (2010), 393-398.

[6] R.S. Young, P.D. Andrew and G.S. Cummings, Effect of simulating leg length inequality on pelvic torsion and trunk mobility, Gate and Posture 11 (2000), 217-223.

[7] K.F. Zabjek, M.A. Leroux and C. Coillard, Acute postural adaptation induced by she lift in idiopathic scoliosis patients, European Spine Journal 10 (2001), 107-113.

[8] B. Gurney, Leg length discrepancy, Gait and Posture 15 (2002), 195-206.

[9] E. Al-Eisa, D. Egan, K. Deluzio and R. Wassersug, Effects of pelvic skeletal asymmetry on trunk movement: threedimensional analysis in healthy individuals versus patients with mechanical low back pain, Spine 31 (2006), E74-79.

[10] M.D. Bussey, Does the demand for asymmetric functional lower body postures in lateral sports relate to structural asymmetry of the pelvis? Journal of Science and Medicine in Sport 13 (2010), 360-364.

[11] M.L. Nault, P. Allard, S. Hinse, L. Blanc, O. Caron, H. Labelle and H. Sadeghi, Relationship between standing stability and body posture parameters in adolescent idiopathic scoliosis, Spine 27 (2002), 1911-1917.

[12] S. Sahli, H. Rebai, S. Ghroubi, A. Yahia, M. Guermazi and M.H. Elleuch, The effects of backpack load and carrying method on the balance of adolescent idiopathic scoliosis subjects, Spine 13 (2013), 1835-1842.

[13] D. Odermatt, P.A. Mathieu, M. Bequsejour, H. Labelle and C.E. Aubin, Electromyography of scoliotic patients treated with a brace, Journal of Orthopaedic Research 21 (2003), 931-936. 
[14] N.M. Hebela and P.J. Tortolani, Idiopathic scoliosis in adults: classification, indications, and treatment options, Spine Surgery 21 (2009), 16-23.

[15] J. Timgren and S. Soinila, Reversible pelvic asymmetry: An overlooked syndrome manifesting as scoliosis, apparent leg length difference, and neurologic symptoms, Journal of Manipulative Physiological Therapeutics 29 (2006), 561-565.

[16] W. Shamberger, The Malalignment Syndrome, Churchill Livingstone, London, 2002.

[17] C.H. Yu, Y.J. Piao, K. Kim and T.K. Kwon, Characteristic analysis of the lower limb muscular strength training system applied with MR dampers, Bio-Medical Materials and Engineering 24 (2014), 297-306.

[18] J.Y. Jung, I.S. Park, B.O. Kim, T.K. Kwon, Y.G. Won and J.J. Kim, Measurement of asymmetric sitting posture using unstable board with accelerometer, International Journal of Biology and Biomedical Engineering 8 (2014), 44-50.

[19] C.H. Yu, S.H. Shin, H.CH. Jeong, D.Y. Go and T.K. Kwon, Activity analysis of trunk and leg muscles during whole body tilt exercise, Bio-Medical Materials and Engineering 24 (2014), 245-254.

[20] W. Dankaerts, P.B. O'Sullivan and A.F. Burnett, Reliability of EMG measurements for trunk muscles during maximal and sub-maximal voluntary isometric contractions in healthy controls and CLBP patients, Journal of Electromyography and Kinesiology 14 (2004), 333-342.

[21] F.J. Vera-Garcia, J.M. Moreside and S.M. McGill, MVC technique to normalize trunk muscle EMG in health women, Journal of Electromyography and Kinesiology 20 (2010), 10-16.

[22] O. Friberg, Clinical symptoms and biomechanics of lumbar spine and hip joint in leg length inequality, Spine 88 (1953), 643-651.

[23] J.P. Gofton, Persistent low back pain and leg length disparity, Journal of Rheumatology 12 (1958), 747-750.

[24] P. van Geffen, J. Reenalda, P.H. Veltink, B.F. Koopman, Decoupled pelvis rotation in sitting: A passive motion technique that regulates development, Journal of Biomechanics 42 (2009), 1288-1294.

[25] A.V. Bruyneel and S. Mesure, Learning effect on the dynamical strategies in sitting position on seesaw motion for idiopathic scoliosis patients, Neurosciene Letters 534 (2013), 264-268.

[26] Z.W. Wang, W.J. Wang, M.H. Sun, Z. Liu, Z.Z. Zhu, F. Zhu, X.S. Qiu, B.P. Qian, S.F. Wang and Y. Qiu, Characteristics of the pelvic axial rotation in adolescent idiopathic scoliosis: a comparison between major thoracic curve and major thoracolumbar/lumbar curve, Spine Journal (2013), In Press.

[27] J. Cheung, A.G. Veldhuizen, J.P. Halberts, W.J. Sluiter and J.R. Van Horn, Geometric and electromyographic assessments in the evaluation of curve progression in idiopathic scoliosis, Spine 31 (2006), 322-329.

[28] P. Mahaudens, M. Raison, X. Banse, M. Mousny and C. Detrembleur, Effect of long-term othotic treatment on gait biomechanics in adolescent idiopathic, Spine, to be published.

[29] V. Guth, F. Abbink and H. Theysohn, Electromyographic investigations on gait: Methods and application in orthopaedics, Electromyography and Clinical Neurophysiology 19 (1979), 305-323. 